\title{
LV-KL
}

\section{International, multicenter, and prospective trials for treatment of HCC}

\author{
Pierce Kah Hoe $\mathrm{CHOW}^{*}$
}

Division of Surgery and Surgical Oncology, Department of Hepato-Pancreato-Biliary and Transplant Surgery, Singapore General Hospital and National Cancer Centre Singapore, Singapore

Lecture: The clinical outcomes of hepatocellular carcinoma still lag behind those of other common cancers such as colorectal and breast cancers. Specifically, there is currently no adjuvant or neoadjuvant therapy in HCC. Pivotal clinical trials are thus needed to address important management issues in HCC.

The last few years has seen positive phase III trials with systemic therapy in advanced HCC including immunotherapy combination. These has thus provided impetus for a range of clinical trials in HCC addressing adjuvant and neoadjuvant therapies in resected HCC, loco-regional therapy combinations in intermediate/locally advanced HCC and systemic combination therapies in advanced HCC. Current pivotal prospective multinational trials for these indications are discussed. 